\title{
c-Jun-mediated repression and transactivation of fibronectin
}

\author{
YUKIO HAYASHI $^{1,2}$, YUJI SHINO ${ }^{1}$, KENGO SAITO $^{1}$, HIDEKI TANZAWA ${ }^{2}$ and HIROSHI SHIRASAWA ${ }^{1}$ \\ Departments of ${ }^{1}$ Molecular Virology, and ${ }^{2}$ Clinical Molecular Biology, Graduate School of Medicine, \\ Chiba University, 1-8-1 Inohana, Chuou-ku, Chiba 260-8670, Japan
}

Received November 5, 2007; Accepted December 3, 2007

\begin{abstract}
Fibronectin (FN) is transactivated by human papillomavirus type 16 (HPV16) E6 via the induction of c-JunATF-2 complexes binding to the cyclic AMP response element (CRE) in the FN promoter. The present study analyzed c-Jun regulation of FN gene expression. Northern and immunoblot analyses showed that c-Jun expression was enhanced in HPV16-E6-expressing cells. However, mouse 10T1/2 cell lines overexpressing c-Jun showed an inverse correlation between the expression levels of c-Jun and those of FN. Luciferase assays indicated that the FN promoter was strongly repressed in c-Jun-overexpressing mouse 10T1/2 cells. Deletion and mutation analyses of the FN promoter revealed that repression of the FN promoter by c-Jun depends on the CRE located at -160 relative to the start site of transcription. Supershift assays of CRE-bound complexes from HPV16-E6-expressing and c-Jun-overexpressing cells suggested that the presence of ATF-2 in the complexes binding to CRE was required for the transactivation of the FN gene. Collectively, our study suggests that the FN gene can be either transactivated or repressed by c-Jun depending on the cell context.
\end{abstract}

\section{Introduction}

Fibronectins (FNs) have critical roles in cell adhesion, migration, differentiation and proliferation via their interactions with integrins and other cell surface receptors. These large glycoproteins are found either in plasma as soluble proteins or in the extracellular matrix in their insoluble form.

Extensive studies have shown that loss of $\mathrm{FN}$ is a common concomitant of transformation (1), and several reports have indicated that the repression of FN function during neoplastic transformation occurs at the transcriptional level (2-4). Accordingly, Werbajh et al identified a 220-bp proximal promoter region involved in the repression of $\mathrm{FN}$ expression (5). The proximal region of the rat FN promoter contains a

Correspondence to: Dr Hiroshi Shirasawa, Department of Molecular Virology (E2), Graduate School of Medicine, Chiba University, 1-8-1 Inohana, Chuou-ku, Chiba 260-8670, Japan

E-mail: sirasawa@faculty.chiba-u.jp

Key words: c-Jun, fibronectin, cyclic AMP response element, human papillomavirus type $16 \mathrm{E} 6$ cyclic AMP response element (CRE) at position -160, a G10 stretch at position -239 and GC boxes at positions -105 and -54 , relative to the transcription start site (4). Oda et al reported that repression of the FN gene during G1-to-S progression is mediated by G10BP, which binds to the G-rich sequences in the FN promoter and prevents SP1 from binding to these sites (6).

We previously showed that the human papillomavirus type 16 E6 (HPV16-E6) protein transcriptionally activates FN, and that modulation of FN expression occurs by E6-induction of binding to the -160 CRE site by a complex containing c-Jun (7). As c-Jun exhibits oncogenic potential to transform cells alone or in the presence of a cooperating oncogene (8-11), transactivation of the FN gene via c-Jun appeared contradictory.

In this study, we examined the mechanism by which c-Jun regulates FN gene expression. Here, we report that overexpression of c-Jun transcriptionally represses FN gene expression via the $-160 \mathrm{CRE}$, and that ATF-2 may be required in the protein complexes bound to CRE for HPV16-E6mediated transactivation of FN.

\section{Materials and methods}

Cells. The 10T/2 mouse cell line was maintained in RPMI1640 medium containing $10 \%$ fetal calf serum (FCS). Cell lines expressing c-Jun were established by transfection of the pRc/CMV2-c-Jun expression plasmid into 10T1/2 cells and subsequent selection with G418. The c-Jun-expressing cell lines and a 10T2/1 cell line stably expressing HPV16-E6, 10E6-3 (12), were maintained in RPMI-1640 medium containing $10 \% \mathrm{FCS}$ and $\mathrm{G} 418(400 \mu \mathrm{g} / \mathrm{ml})$.

Plasmids. pF166Luc, pF123Luc and pF166mLuc were constructed by the HindIII-BglII digestion of rat FN promoter-CAT fusion plasmids, pF166CAT, pF123CAT and pF166mCAT (7), respectively, and the subsequent ligation of the HindIII-BglII fragments into the pGL3-Basic luciferase expression vector (Promega, Madison, WI, USA). To construct the pRc/CMV2-c-Jun expression plasmid, pCMV-c-Jun was digested with EcoRI-BamHI, and the fragment containing mouse c-Jun was ligated into XbaI-HindIII-digested pRc/ CMV2. pCMV-c-Jun and the TAM-67 expression plasmid pCMV-67 were gifts from Michael J. Birrer (13).

Antibodies. Anti-c-Jun, anti-fibronectin, anti-fos, anti-CREB and anti-ATF2 antibodies were purchased from Santa Cruz 
A

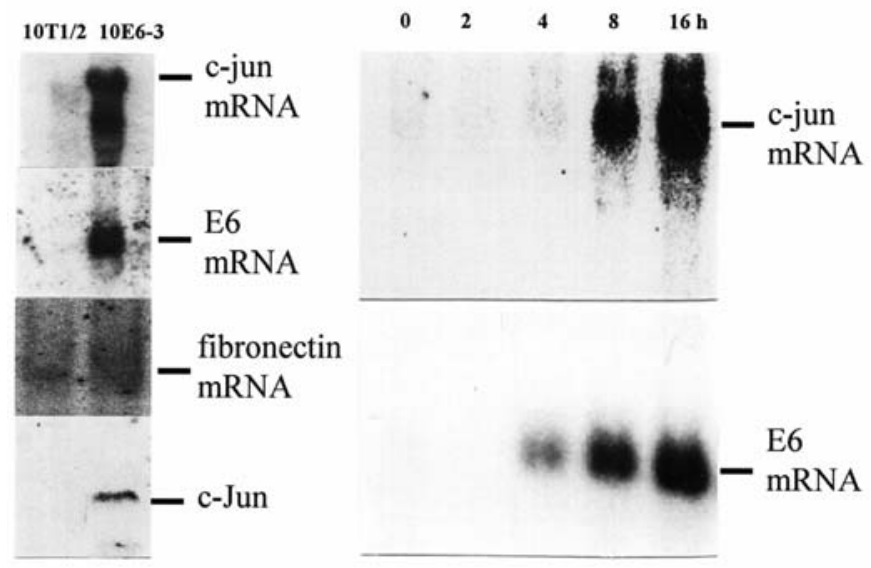

Figure 1. Upregulation of c-Jun expression by HPV16-E6. (A) Northern blot analyses of c-jun mRNA, HPV16-E6 mRNA and fibronectin mRNA, and immunoblot analysis of c-Jun protein. Total mRNA and nuclear extracts from E6-expressing 10T1/2 cells (10E6-3) and parental 10T1/2 cells $(10 \mathrm{~T} 1 / 2)$ were analyzed. Bars indicate the positions of indicated mRNA or protein. (B) Northern blot analyses of c-jun and HPV16-E6 mRNAs in mouse 10T1/2 cells infected with Adex16E6. Total-RNA was extracted from cells at the indicated times $(0,2,4,8$ and $16 \mathrm{~h})$ after infection with E6expressing recombinant adenovirus (Adex16E6). Bars indicate the positions of c-jun and HPV16-E6 mRNAs.

Biotechnology (Santa Cruz, CA, USA) or Transduction Laboratories (Lexington, KY, USA).

Northern blot analysis. Total-RNA was extracted via the acid guanidinium thiocyanate-phenol-chloroform method (Chomzynskin). Purified RNA samples were separated electrophoretically (20 $\mu \mathrm{g}$ per lane) and transferred onto a polyvinylidene difluoride filter (Hybond-XL, Amersham Pharmacia Biotec). Hybridizations were performed under stringent conditions, as described previously (14). mRNA expression levels were quantified using a BAS 2500 Bioimaging analyzer (Fujix, Japan).

Western blot analysis. Cell extracts were mixed with a buffer containing $25 \mathrm{mmol} /$ Tris- $\mathrm{HCl}, 1 \%$ sodium dodecyl sulfate (SDS), $5 \%$ glycerol, $1 \%$ 2-mercaptoethanol and $0.1 \%$ bromophenol blue, and boiled for $5 \mathrm{~min}$. The samples were resolved on $12.5 \%$ SDS polyacrylamide gels and transferred electrophoretically onto filters (Immobillon, Millipore, Bedford, MA). The membranes were treated with $10 \%$ non-fat dry milk in PBS containing $0.01 \%$ Tween-20 (PBS-T) and incubated with anti-c-Jun, anti-fibronectin or anti-ATF-2 antibody for $6 \mathrm{~h}$ at $4^{\circ} \mathrm{C}$. Blots were washed with PBS-T, incubated with horseradish peroxidase-conjugated secondary antibody for $1 \mathrm{~h}$, processed with the ECL Plus Detection Kit (Amersham Pharmacia Biotech, Buckinghamshire, UK) and exposed to film.

Luciferase reporter assays. Cells were transfected with $5 \mu \mathrm{g}$ of luciferase reporter plasmid mixed with $1 \mu \mathrm{g}$ of the pRL-SV40 internal marker (Promega). After 48 h, cells were washed twice with PBS, collected by scraping and freeze-thawed three times. The lysates were analyzed for luciferase activity with a Wallac 1420 ARVOsx (Perkin Elmer) using the Dual-
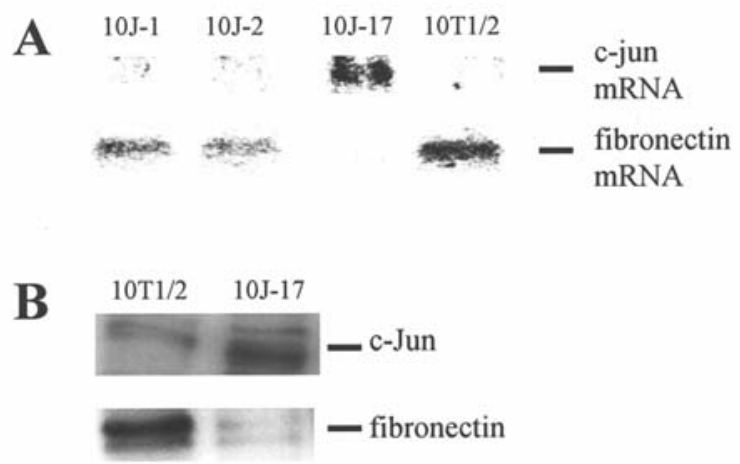

Figure 2. Expression of c-jun and fibronectin in c-Jun-expressing cell lines. (A) Northern blot analyses of c-jun and fibronectin mRNAs. Total-RNA was extracted from c-jun-expressing cell lines (10J-1, 10J-2, 10J-17) and parental 10T1/2 cells, and subjected to Northern blot analyses. Bars indicate the positions of c-jun and fibronectin mRNAs. (B) Immunoblot analyses of c-Jun and fibronectin. Protein extracts $(20 \mu \mathrm{g})$ from 10T1/2 and c-junexpressing cells were subjected to immunoblot analyses. Bars indicate the positions of c-Jun and fibronectin.

luciferase assay (Promega), according to the manufacturer's instructions. Experiments were repeated three times.

Recombinant adenovirus. Adex16E6 is a recombinant adenovirus that expresses HPV16-E6 (nt. 94-571) without the splice donor site $(15,16)$. For transient expression of HPV16-E6, cells were infected with Adex16E6 at an MOI of 100.

Nuclear extracts and gel retardation assays. Nuclear extracts were prepared from rapidly growing subconfluent cultures as described previously (7). All extracts were standardized for protein concentration before use. The ${ }^{32} \mathrm{P}$-labeled DNA fragment used as a probe was obtained by Klenow enzyme filling with $\left[\alpha-{ }^{32} \mathrm{P}\right] \mathrm{dATP}$. Binding reactions were carried out in $20-\mu 1$ reaction volumes as described previously (7). For antibody supershift assays, specific polyclonal antibodies to c-Jun, ATF-2, CREB or Fos, were added to the binding reaction mixture prior to the addition of the labeled oligo-nucleotide for $2 \mathrm{~h}$ at $4^{\circ} \mathrm{C}$. Binding reaction mixtures were incubated for $30 \mathrm{~min}$ at room temperature. DNA-protein complexes were separated from unbound DNA by electrophoresis in $5 \%$ polyacrylamide gels.

\section{Results}

Induction of c-Jun by HPV16-E6. We previously showed that HPV16-E6 protein transcriptionally modulates FN gene expression via $\mathrm{CRE}$ by inducing the binding of protein complexes consisting of c-Jun and ATF-2 to CRE sites (7). To further investigate the role of c-Jun in FN gene expression, we first examined the effect of HPV16-E6 on the expression level of c-Jun. Consistent with previous findings, FN mRNA expression in the HPV16-E6-expressing 10E6-3 mouse cell line was enhanced compared to the $10 \mathrm{~T} 1 / 2$ parental cell line (Fig. 1A). Notably, both c-Jun mRNA and c-Jun protein were highly expressed in 10E6-3 cells compared to control cells. To test if c-Jun is transactivated by HPV16-E6, 10T1/2 cells were infected with the recombinant Adex16E6 adenovirus, which expresses HPV16-E6 (16). As shown in Fig. 1B, the expression 
A
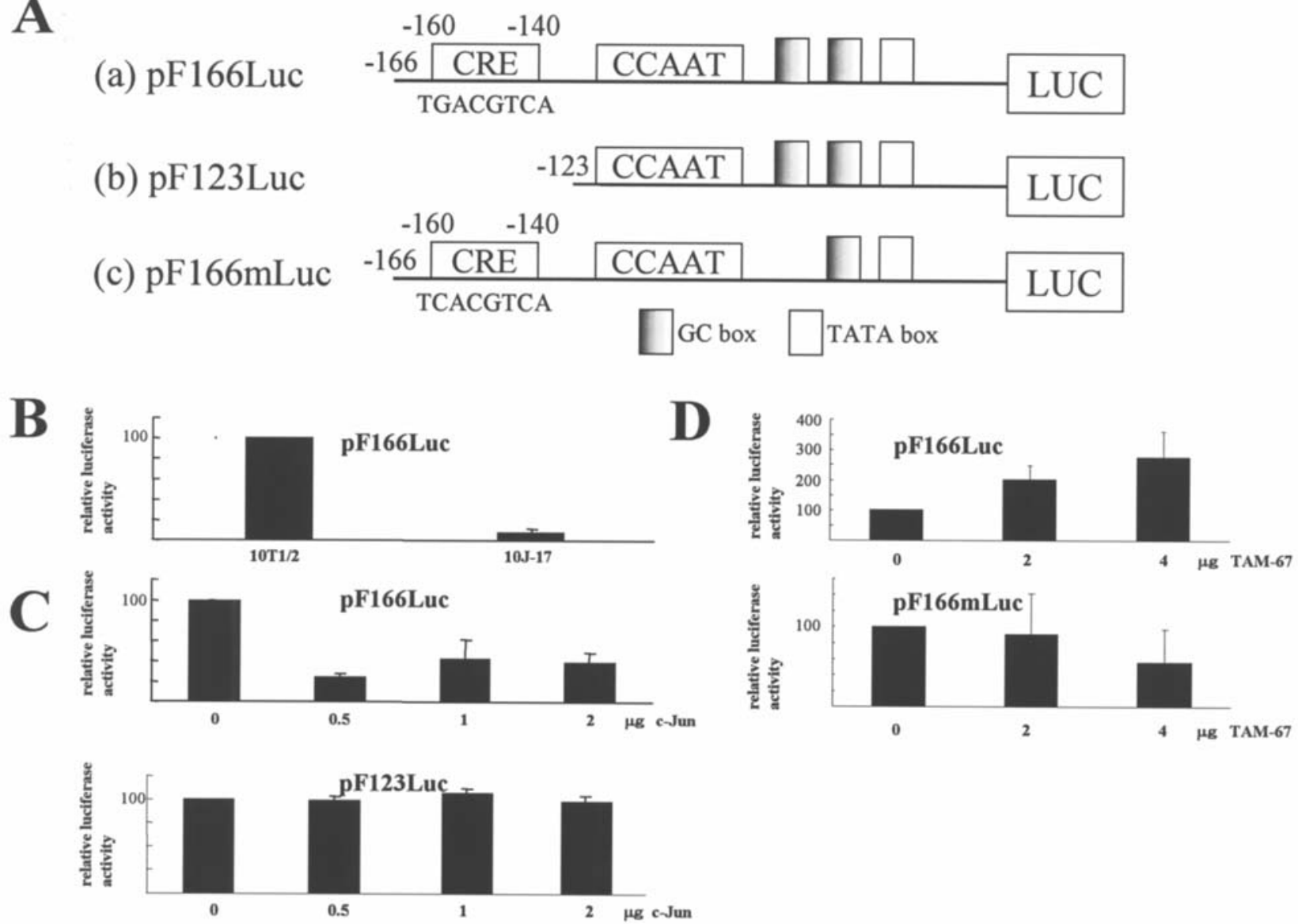

Figure 3. Repression of the fibronectin promoter by overexpression of c-Jun. (A) Schematic representation of fibronectin promoter-luciferase fusion plasmids. (a) pF166Luc contains CRE (TGACGTCA) at position -160, CCAAT box at position -140, GC boxes at position -105 and -54 and a TATA box at position -30 relative to the start site of transcription. (b) pF123Luc lacks the -160 CRE and -140 CCAAT box. (c) pF166mLuc contains a mutated -160 CRE (TCACGTCA). (B) Luciferase activity of the pF166Luc in 10T1/2 and c-jun-expressing 10J-17 cells. pF166Luc (5 $\mu \mathrm{g})$ was transfected into $10 \mathrm{~T} 1 / 2$ and $10 \mathrm{~J}-17$ cells, along with $1 \mu \mathrm{g}$ of pRL-SV40 as an internal control. The relative luciferase activity in $10 \mathrm{~T} 1 / 2$ cells calculated as 100 is the mean of three independent experiments. The error bar indicates the standard error. (C) Effects of c-Jun on fibronectin promoter activity. Five micrograms of pF166Luc (upper) or pF123Luc (lower) were co-transfected into 10T1/2 cells with increasing amounts of pCMV-c-Jun expression plasmid (0, 0.5, 1 and $2 \mu \mathrm{g})$ and luciferase activity was determined $24 \mathrm{~h}$ after transfection. The relative luciferase activities are the means of three independent experiments. (D) Effects of the dominantnegative mutant of c-Jun, TAM-67, on fibronectin promoter activity. Five micrograms of pF166Luc (upper) or pF166mLuc were co-transfected into 10T1/2 cells with increasing amounts of the pCMV-67 TAM67 expression plasmid (0,2 and $4 \mu \mathrm{g})$ and luciferase activity was determined $24 \mathrm{~h}$ after transfection. The relative luciferase activities are the means of three independent experiments. Error bars indicate standard errors.

of c-jun mRNA was enhanced $8 \mathrm{~h}$ after infection with Adex16E6, following increased expression of E6. Infection with the empty adenovirus vector had no effect on the expression of c-Jun (data not shown). These data suggested that, in addition to the induction of binding of c-Jun-containing protein complexes to CRE, HPV16-E6 may also enhance FN gene expression through the stimulation of c-Jun expression.

Repression of endogenous FN gene expression by overexpression of c-Jun. To examine the effect of c-Jun overexpression on FN gene expression, we established a panel of mouse cell lines constitutively overexpressing c-Jun by transfecting pRc/CMV2-c-Jun into mouse 10T1/2 cells and selecting with G418. Several cell lines, designated as 10J-1, $10 \mathrm{~J}-2$ and $10 \mathrm{~J}-17$, were established and subsequently characterized. As shown in Fig. 2A, we detected an inverse correlation between the expression levels of c-Jun and FN in the c-Junexpressing cell lines. High levels of c-jun mRNA were observed in 10J-17 cells, and weak levels in 10J-1 and 10J-2 cells. In contrast, FN mRNA expression was strongly decreased in $10 \mathrm{~J}-17$ cells and weakly repressed in $10 \mathrm{~J}-1$ and $10 \mathrm{~J}-2$ cells compared to parental 10T1/2 cells (Fig. 2A). Consistent with results from the mRNA analysis, the downregulation of $\mathrm{FN}$ protein expression and the enhancement of c-Jun protein expression in the c-Jun-overexpressing $10 \mathrm{~J}-17$ cells compared to the parental 10T1/2 cells was confirmed (Fig. 2B).

Repression of the FN promoter by overexpression of c-Jun. To examine whether the decreased level of FN occurs at the transcriptional level, 10J-17 and 10T1/2 cells were transfected with $\mathrm{pF} 166 \mathrm{Luc}$ luciferase reporter driven by FN promoter (Fig. 3A). Luciferase activity in $10 \mathrm{~J}-17$ cells was only $5 \%$ relative to that of the $10 \mathrm{~T} 1 / 2$ cells, indicating a strong repression of FN promoter activity and suggesting that repression of $\mathrm{FN}$ gene expression occurs at the transcriptional level in c-Jun-overexpressing 10J-17 cells (Fig. 3B).

To confirm whether c-Jun represses the FN promoter, $10 \mathrm{~T} 1 / 2$ cells were co-transfected with $\mathrm{pF} 166 \mathrm{Luc}$ and increasing 


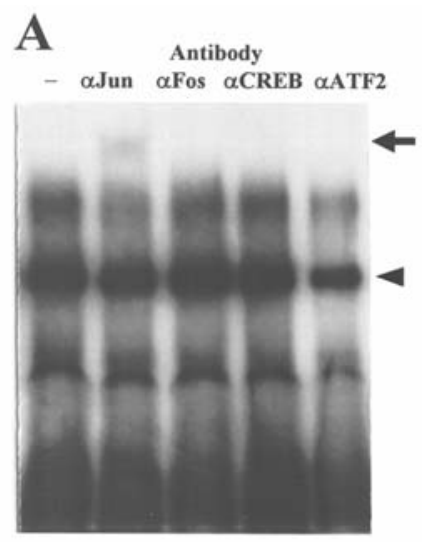

10T1/2/Adex16E6

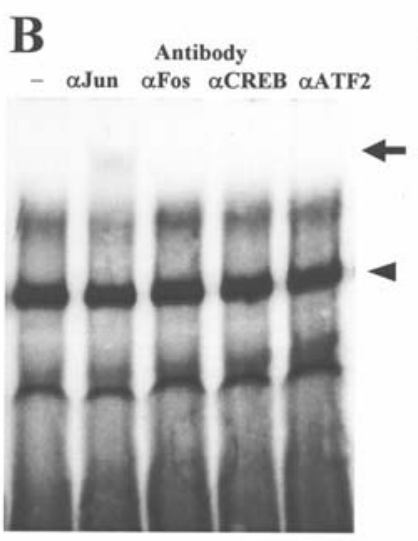

$10 \mathrm{~J}-17$
C

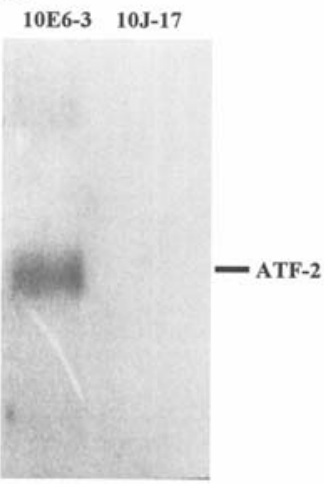

Figure 4. Complexes binding to CRE in HPV16-E6 and c-Jun-overexpressing cells. Antibody supershift assays of protein complexes bound to CRE in extracts from 10T1/2 cells infected with HPV16-E6 adenovirus (A) and c-jun-overexpressing 10J-17 cells (B). Antibodies specific to c-Jun ( $\alpha$ Jun), c-Fos ( $\alpha$ Fos), CREB $(\alpha \mathrm{CREB})$ and ATF-2 $(\alpha \mathrm{ATF} 2)$ were added to the binding reaction mixture $2 \mathrm{~h}$ prior to the addition of the labeled probe and incubated at $4{ }^{\circ} \mathrm{C}$. The arrowhead denotes the specific complexes predicted, and the arrow indicates the supershifted band. (C) Immunoblot analyses of ATF-2. Protein extracts (20 $\mu \mathrm{g}$ ) from HPV16-E6-expressing 10T1/2 cells (10E6-3) and c-jun-overexpressing cells (10J-17) were subjected to immunoblot analyses. The bar indicates the position of ATF-2.

amounts of the pCMV-c-Jun expression plasmid, and luciferase activity was assessed. The transient expression of c-Jun efficiently repressed the activity of pF166Luc; notably, no effect was observed using pF123Luc lacking CRE at -160 (Fig. 3C), suggesting that $-160 \mathrm{CRE}$ is necessary for c-Junmediated repression of the FN promoter.

TAM-67, a deletion mutant of c-Jun lacking the transactivation domain and retaining DNA binding activity, competes and inhibits c-Jun transcriptional activation by preventing wildtype c-Jun from binding to CRE (13). To examine the effect of TAM-67 on the activity of the FN promoter, pF166Luc was co-transfected into $10 \mathrm{~T} 1 / 2$ cells along with the TAM-67 expression plasmid pCMV-67. Under these conditions, a dose-dependent increase in luciferase activity was observed (Fig. 3D) and mutation of the CRE site (pF166mLuc) abolished the effects of pCMV-67.

Complexes binding to CRE in c-Jun-expressing cells. In a previous study (7), we showed that nuclear extracts from the 10E6-3 E6-expressing mouse cells exhibited greater DNA binding activity of protein complexes to the CRE site, and that these complexes contained c-Jun and ATF-2. To confirm that transiently expressed E6 promotes c-Jun/ATF-2 DNA binding to the consensus CRE site, whole cell extracts from 10T1/2 cells infected with Adex16E6 were examined for DNA binding activity. As shown in Fig. 4A, the CRE-protein complex band was supershifted by the anti-c-Jun antibody, while the anti-ATF-2 antibody reduced binding dramatically ( $\sim 3$-fold reduction in band intensity); the bands were neither supershifted nor disrupted by either the anti-c-Fos or antiCREB antibodies. However, in 10J-17 cells that overexpressed c-Jun and lacked HPV16-E6 expression, the DNA-protein complex was supershifted by anti-c-Jun but not disrupted by the anti-ATF2 antibody (Fig. 4B), indicating that the CREbound protein complexes lacked ATF-2. Immunoblot analyses showed that ATF-2 expression was observed in 10E6-3 but not 10J-17 cells (Fig. 4C), supporting the absence of ATF-2 in the CRE-bound protein complexes of $10 \mathrm{~J}-17$ cells.

\section{Discussion}

Our study demonstrated that c-Jun both transactivates and represses FN gene expression depending on the cellular context. Decreased FN production is often observed following oncogenic transformation, leading to decreased adhesion and increased metastatic potential $(1,5)$. Conversely, restoration of FN expression has been shown to revert tumorigenic and/or metastatic phenotypes (17). However, it has also been reported that FN gene expression is upregulated in transformed cells by several oncoproteins $(18,19)$.

The human FN promoter contains three CREs, one of which is bound by the c-Jun-ATF-2 heterodimer. TGF- $\beta$ mediates FN synthesis by modulating the activity of c-Jun-ATF-2 via the CRE of the FN promoter (20). In a previous report, we demonstrated that the oncoprotein HPV16-E6 transactivates the FN gene, inducing the binding activity of c-Jun-ATF-2 complexes to CRE (7). The current study suggests that the FN gene repression often observed in cancer cells could be modulated or de-repressed depending on the cellular context through the course of tumor progression.

This study also revealed that HPV16-E6 not only transactivates FN gene expression, but also c-Jun expression. Furthermore, the overexpression of c-Jun in the absence of HPV16-E6 expression repressed FN gene expression. Downregulation of FN gene expression in c-Jun-overexpressing cells, in which CRE-bound protein complexes lacked ATF-2, indicated that ATF-2 expression and CRE binding could be required for the transactivation of FN gene expression.

Transactivation of the FN gene by the dominant-negative c-Jun TAM-67 in 10T1/2 cells that express low levels of ATF-2 suggested that c-Jun complexes lacking ATF-2 can mediate repression. In our study, upregulation of the $\mathrm{FN}$ gene by TAM-67 could possibly be due to TAM-67 releasing the endogenous c-Jun-mediated repression.

The protein complexes involved in the repression of the $\mathrm{FN}$ gene could be mediated by either c-Jun homodimers or c-Jun heterodimers with unknown factors besides ATF-2. Our study 
suggests that the same transcription regulatory sequence could tightly regulate both the transactivation and repression of FN, depending on the cellular context. This hypothesis would explain the complicated expression pattern of the FN gene in cancer cells, and understanding the molecular mechanism by which the regulation of $\mathrm{FN}$ is disrupted and expression is repressed during tumorigenesis warrants further investigation.

\section{References}

1. Hynes RO and Yamada KM: Fibronectins: multifunctional modular glycoproteins. J Cell Biol 95: 369-377, 1982.

2. Fagan JB, Sobel ME, Yamada KM, De Crombrugghe B and Pastan I: Effects of transformation on fibronectin gene expression using cloned fibronectin cDNA. J Biol Chem 256: $520-525,1981$

3. Jochemsen AG, Bernards R, van Kranen HJ, Houweling A, Bos JL and van der Eb AJ: Different activities of the adenovirus types 5 and 12 E1A regions in transformation with the EJ Ha-ras oncogene. J Virol 59: 684-691, 1986.

4. Nakamura T, Nakajima T, Tsunoda S, Nakada S, Oda K, Tsurui H and Wada A: Induction of E1A-responsive negative factors for transcription of the fibronectin gene in adenovirus E1transformed rat cells. J Virol 66: 6436-6450, 1992.

5. Werbajh SE, Urtreger AJ, Puricelli LI, De Lustig ES, Bal de Kier Joffe E and Kornblihtt AR: Downregulation of fibronectin transcription in highly metastatic adenocarcinoma cells. FEBS Lett 440: 277-281, 1998.

6. Oda E, Shirasuna K, Suzuki M, Nakano K, Nakajima T and Oda K: Cloning and characterization of a GC-box binding protein, G10BP-1, responsible for repression of the rat fibronectin gene. Mol Cell Biol 18: 4772-4782, 1998.

7. Shino Y, Shirasawa H, Kinoshita T and Simizu B: Human papillomavirus type 16 E6 protein transcriptionally modulates fibronectin gene expression by induction of protein complexes binding to the cyclic AMP response element. J Virol 71: 4310-4318, 1997.

8. Schutte J, Minna JD and Birrer MJ: Deregulated expression of human c-jun transforms primary rat embryo cells in cooperation with an activated c-Ha-ras gene and transforms rat-1a cells as a single gene. Proc Natl Acad Sci USA 86: 2257-2261, 1989.
9. Eferl R and Wagner EF: AP-1: a double-edged sword in tumorigenesis. Nat Rev Cancer 3: 859-868, 2003.

10. Vogt PK and Bader AG: Jun: stealth, stability, and transformation. Mol Cell 19: 432-433, 2005.

11. Heasley LE and Han SY: JNK regulation of oncogenesis. Mol Cell 21: 167-173, 2006.

12. Shimizu K, Shirasawa H, Jin M-H, Inaba N, Sekiya S and Simizu B: Alternative splicing of human papillomavirus type-16 E6-mRNA in mouse and monkey cell lines. Int $\mathrm{J}$ Oncol 4: 971-976, 1994.

13. Brown PH, Chen TK and Birrer MJ: Mechanism of action of a dominant-negative mutant of c-Jun. Oncogene 9: 791-799, 1994.

14. Shirasawa H, Tomita Y, Kubota K, Kasai T, Sekiya S, Takamizawa H and Simizu B: Transcriptional differences of the human papillomavirus type 16 genome between precancerous lesions and invasive carcinomas. J Virol 62: 1022-1027, 1988

15. Shirasawa H, Jin MH, Shimizu K, Akutsu N, Shino Y and Simizu B: Transcription-modulatory activity of full-length E6 and E6*I proteins of human papillomavirus type 16. Virology 203: 36-42, 1994.

16. Sashiyama H, Shino Y, Kawamata Y, Tomita Y, Ogawa N, Shimada H, Kobayashi S, Asano T, Ochiai T and Shirasawa H: Immortalization of human esophageal keratinocytes by E6 and E7 of human papillomavirus type 16. Int J Oncol 19: 97-103, 2001.

17. Yamada KM, Yamada SS and Pastan I: Cell surface protein partially restores morphology, adhesiveness, and contact inhibition of movement to transformed fibroblasts. Proc Natl Acad Sci USA 73: 1217-1221, 1976.

18. Allen-Hoffmann BL, Schlosser SJ, Brondyk WH and Fahl WE: Fibronectin levels are enhanced in human fibroblasts overexpressing the c-sis protooncogene. J Biol Chem 265: 5219-5225, 1990.

19. Sheibani N, Rhim JS and Allen-Hoffmann BL: Malignant human papillomavirus type 16-transformed human keratinocytes exhibit altered expression of extracellular matrix glycoproteins. Cancer Res 51: 5967-5975, 1991.

20. Hocevar BA, Brown TL and Howe PH: TGF-beta induces fibronectin synthesis through a c-Jun $\mathrm{N}$-terminal kinasedependent, Smad4-independent pathway. EMBO J 18: 1345-1356, 1999. 